\title{
Review on Pharmacologically Active Metabolites from Yarsagumba (Ophiocordyceps sinensis), an Epitome of Himalayan Elixir
}

\author{
S. Shrestha ${ }^{1,5}$, A. K. Shrestha ${ }^{2}$, J.H. Park ${ }^{1}$, D. Y. Lee ${ }^{3}$, J. G. Cho ${ }^{1}$, B. Shrestha ${ }^{4,5}$ \\ and N. I. Baek ${ }^{1}$ \\ ${ }^{1}$ Graduate School of Biotechnology, Institute of Life Sciences and Resources, Kyung Hee \\ University, Yongin 446-701, Republic of Korea \\ ${ }^{2}$ G.P.O. Box 6133, Kathmandu, \\ ${ }^{3}$ Department of Medicinal Crop Research, National Institute of Horticultural and Herbal \\ Science, Rural Development Administration, Eumseong 369-873, \\ Republic of Korea \\ ${ }^{4}$ Mushroom Research Division, National Institute of Horticultural and Herbal Science, Rural \\ Development Administration, Eumseong 369-873, Republic of Korea \\ ${ }^{5}$ Green Energy Mission/Nepal, Kathmandu \\ e-mail:nibaek@khu.ac.kr
}

\begin{abstract}
Yarsagumba (Ophiocordyceps sinensis (Berk.) Sung et al.) is a well-known entomogenous fungus native to alpine nival terrain of trans-Himalaya and Tibetan Plateau. The traditional use of $O$. sinensis in Ayurvedic medicine as well as in traditional Chinese medicine for range of human health requirements with well documented evidences are themselves testimony of its value. It is regarded as winter worm, summer grass due to its association with Thitarodes (Hepialus) larvae. The extremities of habitat condition and endurance of fungus to sustain adversaries by production of metabolites has led to unique profile of metabolites including nucleosides, proteins and nitrogenous compounds, polysaccharides, sterols, fatty acids and their derivatives, as well as some vitamins and inorganics. There are wide ranges of biological activities that have been reported from $O$. sinensis including anti-inflammatory, antioxidant, anti-tumor, anti-metastatic, immunomodulatory, antimicrobial, insecticidal, hypolipidaemic, hypoglycemic, antiageing, lipolytic, neuroprotective, renoprotective effects, etc. Although several components can be responsible for activity of compounds, understanding ultimate compounds which fit with biomolecular target is crucial to combat diseases and development of new class of effective drugs. In this review a concise evaluation of pharmacological activities of metabolites reported for $O$. sinensis are done to provide insight into its biologically active components.
\end{abstract}

Key words: active metabolite, bioactivity, Cordyceps sinensis, pharmacology

\section{Introduction}

Ophiocordyceps sinensis (Berk.) Sung et al. (Ophiocordicipitaceae, Hypocreales, Ascomycota, Kingdom Fungi), the most valued among fungi, is distributed in trans-Himalayan terrain of Nepal, Tibetan Plateau, India and Bhutan in the altitudinal range of
3,000 to 5,000 asl. On the basis of phylogenetic study (Sung et al. 2007), Ophiocordyceps sinensis was named for Cordyceps sinensis (Berk.) Sacc. It is popularly known as Yarsagumba in Nepal. It is also referred as Jivan Buti, Kira Chhyau, Kira Jhar, Saram 
Buti, Jingani etc in Nepal (Shrestha et al. 2010). It is regarded as highly effective for several human maladies including cancer, hypoglycemia, asthma, hypercholesterolaemia, sexual dysfunction, immunodeficiency, etc (Zhu et al. 1998a,b). Further, Holliday and Cleaver (2008) reported several pharmacological activities including improvement of physical performance, circulatory functions, hepatoprotection, renoprotection, atherosclerosis, anti-tumor and anti-metastatic. Another important aspect is its use as tonic, aphrodisiac, cardiotonic and expectorant (Baral \& Kurmi 2006) and its market value can even reach US\$ 800 for an ounce (Giove 2011). Traditionally in Nepal, powdered $O$. sinensis is taken along with honey, milk, water or its extract in an alcoholic drink (Devkota 2006). There is also report of administration of its infusion with powdered root of Dactylorhiza hatagirea (Paanch aunle) or Ephedra gerardiana (Somlata) (Gewali 2008). The scientific name $O$. sinensis refers to the sexual stage including stalked fruiting-body whereas the asexual mycelium culture is termed as Hirsutella sinensis (Shrestha et al. 2010). The fungal invasion in host larva and its growth occur in late summer, and its growth eventually leads to replacement of internal organs leading to formation of thickened fungal tissue known as endosclerotium during winter (Chen et al. 2004); whereas fruiting-body (stroma) sprouts from the prethorax region of infected larvae and matures in late summer (June-August) (Li \& Yang 2009). There are about 60 taxa as potential hosts of $O$. sinensis mostly belonging to genus Thitarodes (Hepialus) (Wang \& Yao 2011). They are commonly known as bat-moths, swift-moths or ghost-moths which depend on soil humus and tender roots of Ranunculus brotherusii, Cyananthus macrocalyx, Juncus leucanthus and Veronica ciliate etc (Lei et al. 2011). The entire funguslarva combination is collected for medicinal use. The colour of $O$. sinensis varies from light brown to brown or black depending on habitat condition. The distribution limit, and ever increasing demands have prompted the isolation of mycelium strains and largescale culture. As cost involved in getting wild specimens is tremendous, the majority of researches on $O$. sinensis are based on mycelium culture and some similarity in effectiveness and content have been reported for both wild and cultured $O$. sinensis (Li et al. 2002). However, the charm of natural $O$. sinensis remains intact and there is a billion dollar trade in practice. The question what is present in $O$. sinensis which makes it so popular or effective has still not been answered exactly although there have been several researches to find out the cause. Several biological activities are based on extract, fractions and polysaccharides and there are discrepancies in structure determination of polysaccharides (Chen et al. 2013). The fruit-body production in cultivation has not been successful yet and mycelia culture is the major source of studies (Dong \& Yao 2011). In comparison to cultured mycelium, the natural $O$. sinensis undergoes cascade of events from the selection of host up to the formation of fruiting enduring extreme environmental conditions together with interaction with Cordyceps-associated species. There is great likelihood of presence of active small molecule compounds in wild $O$. sinensis but isolation and identification of such compounds are still awaited due to enormous cost of wild harvest. There is also equal chance of synergetic effect of several compounds responsible for some of the activities. The recent report of the major pharmacological activities of $O$. sinensis and a comprehensive summary compounds responsible are reviewed here which are presented as shaded matrix to elucidate possible active components.

The major metabolites include proteins and nitrogenous compounds, polysaccharides, sterols, nucleosides, fatty acids and their derivatives, vitamins and inorganics (Zhu et al. 1998a,b, Holliday \& Cleaver 2008). The nucleosides are regarded as the active components and adenosine has been used as chemical marker for quality control of $O$. sinensis (Shiao et al. 1994). In addition to adenosine, Li et al. (2006a) reported other marker compounds of wild $O$. sinensis as ergosterol, adenosine, cordycepin, guanosine, inosine, uridine, mannitol and polysaccharides which have been reported for their biological activities (Zhao et al. 2013). The relatively higher amount of inosine $(0.33 \mathrm{mg} / \mathrm{g})$, ergosterol (3.65-10.34 mg/g), mannitol $(38.64-35.42 \mathrm{mg} / \mathrm{g}$ ) in natural $O$. sinensis in comparison to cultured ones producing $0.03-0.19 \mathrm{mg} / \mathrm{g}$ of inosine, $0.38-1.31 \mathrm{mg} / \mathrm{g}$ of ergosterol, $10.24-13.41 \mathrm{mg} / \mathrm{g}$ of mannitol are noteworthy (Li et al. 2006a). One of the active compounds, cordycepin (13), which is a major component of Cordyceps militaris (Wang et al. 2009a), was reported in small quantity $(0.04-0.06 \mathrm{mg} / \mathrm{g})$ in wild but absent in cultured $O$. sinensis ( $\mathrm{Li}$ et al. 2006a). However, Kim \& Yun (2005) reported 18.19 mg/l cordycepin in cultured $O$. sinensis and mentioned 
variation in cordycepin content depending on strain. Some other noteworthy constituents in O. sinensis are carbohydrates, D-mannitol, trehalose, uridine, adenine, adenosine and guanosine (Wang et al. 2009a). Chen et al. (2009) reported two new epipolythio dioxopiperazines, gliocladicillins A, B and 11,112 -dideoxyverticillin from Cordyceps-associated fungi which indicated possibly beneficial $O$. sinensis associated species. Yang et al. (2011) isolated 50 compounds, including five new constituents, cordysinins A-E. Yu et al. (2012) reported fifty-one volatile compounds including aldehydes, alcohols, ketones, esters, aromatics, phenols, acids, alkanes, alkenes, furans and other phenolic compounds from the mycelia cultured by solid-state media and submerged fermentation. Although there are several possibilities of synergetic relation of compounds working to give some activities, the lock-and-key theory and one hit target to combat certain disease is required in practice for effective use of well defined drugs, their production and commercialization for the benefit of mass population.

\section{Dose and toxicity}

The acute toxicity in bone marrow chromosome aberrations and Ames test showed $O$. sinensis as safe (Shen et al. 2001). Lie et al. (2005) reported non toxic nature of polysaccharide from $O$. sinensis in test for animal mass, biochemical properties of blood, and histopathological changes of liver and kidney. Further, for median lethal does $\left(\mathrm{LD}_{50}\right)$, mice injected up to $80 \mathrm{~g} / \mathrm{kg}$ per day, did not show any fatalities after 7 days of treatment (Holliday et al. 2005). Meena et al. (2013) also highlighted on non toxic nature of laboratory cultured mycelia of $O$. sinensis oral administration up to $2 \mathrm{~g} / \mathrm{kg}$ body weight in adult female Wister rats. The human equivalent dose (HED) of $20 \mathrm{mg} /$ $\mathrm{kg}$ in mice is $1.62 \mathrm{mg} / \mathrm{kg}$ calculated on the basis of body surface area, or it is equivalent to $113 \mathrm{mg}$ for $70 \mathrm{~kg}$ person (Liu et al. 2006). There are very few reports of toxic side effects like dry mouth, nausea or diarrhea (Zhu et al. $1988 \mathrm{a}, \mathrm{b})$. Rabbit given $10 \mathrm{~g} /$ $\mathrm{kg}$ per day $(\mathrm{n}=6)$ did not show any abnormalities in blood test or in kidney or liver function but reported a case of systemic allergic reaction after taking a strain of Cs-4 (Huang et al. 1987). However, no published data are available on effect on children as well as pregnant or lactating women. Intake of $6 \mathrm{~g}$ per day of $O$. sinensis has been reported for therapeutic purpose in treatment of cancer (Holliday \& Cleaver 2008).

The quality control and authentication of $O$. sinensis product is one of the major issues for safety and pharmacological efficacy. The uses of multiple chemical markers such as nucleosides, ergosterol, mannitol and polysaccharides used for quality control also have some drawbacks (Li et al. 2006a). Fu et al. (2011) reported process for more precise authentication of sample continuous monitoring of cellular impedance in real time, which produces specific time/dose dependent cell response profiles (TCRPs) in addition to spectroscopic fingerprinting of active compounds by HPLC using adenosine (or 3-deoxyadenosine) as a standard, in accordance with Pharmacopoeia of the People's Republic of China (2005 version). The use of bar-coding and fingerprinting to some extent are in practice now-a-days with accession of ITS and 18s ribosomal RNA gene (MMDBD 2012).

\section{Pharmacologically active metabolites}

The chemical constituents profile shows variation in natural and cultured $O$. sinensis. Guan et al. (2010) reported occurrence of more than $7.99 \%$ free mannitol in natural but less than $5.83 \%$ mannitol in cultured $O$. sinensis. Zhu et al. (1998a) classified seven classes of chemical constituents in natural and mycelium fermentation of $O$. sinensis as sterols, nucleosides, sugar derivatives, fatty acids, organic acids, proteins and vitamins. Shiao et al. (1994) reported similarity in nucleoside patterns in fruiting body and cultured mycelium and also reported presence of significant amount of adenosine $(2.47 \mathrm{mg} / \mathrm{g})$ in fruiting bodies. There can be variation in constituents of $O$. sinensis depending on the place of origin and type of strain used for mycelium culture. In traditional Chinese medicine practice, the therapeutic effects reported for $O$. sinensis are due to contribution of multiple components. There exist extensive reviews on clinical aspects of $O$. sinensis (Zhu et al. 1998a,b, Holliday 2008, Li \& Yang 2009, Wang et al. 2012 b) as well as on activity of extracts (Lin \& Li 2011). In previous review (Shrestha et al. 2012) we provided overview of compounds reported for O. sinensis. As there are limited reviews specifically dealing with pharmacologically active metabolites, in this brief review we have tried to summarize major effective components that have been reported for O. sinensis (Matrix 1). 
Nepal Journal of Science and Technology Vol. 14, No. 2 (2013) 49-58

Matrix 1. Shaded matrix display of major pharmacological activities of compounds from Ophiocordyceps sinensis*

\begin{tabular}{|c|c|c|c|c|c|c|c|c|c|c|c|c|}
\hline \multirow[b]{2}{*}{ Compounds } & \multicolumn{12}{|c|}{ Pharmacological Activities } \\
\hline & 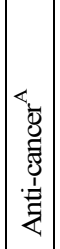 & 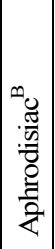 & 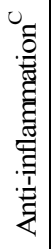 & 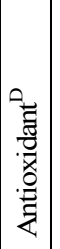 & 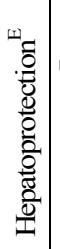 & 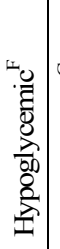 & 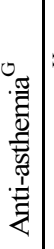 & 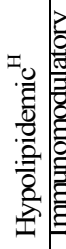 & 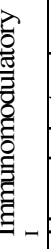 & 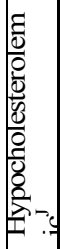 & 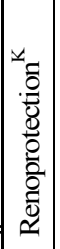 & 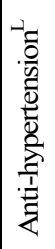 \\
\hline \multicolumn{13}{|l|}{ ergosterol (1) } \\
\hline \multicolumn{13}{|l|}{ ergosterol peroxide (2) } \\
\hline \multicolumn{13}{|c|}{$5 \alpha, 8 \alpha$-epidioxy-22- $E$-ergosa-6,9(11), 22-trien-3 $\beta$-ol (3) } \\
\hline \multicolumn{13}{|c|}{ ergosteryl-3- $O$ - $\beta$-D-glucopyranoside (4) } \\
\hline \multicolumn{13}{|c|}{$\begin{array}{l}5 \alpha, 8 \alpha \text {-epidioxy-24( } R) \text {-methylcholesta-6,22-dien-3 } \beta \text {-D- } \\
\text { glucopyranoside (5) }\end{array}$} \\
\hline \multicolumn{13}{|c|}{$(3 \beta, 5 \alpha, 6 \beta, 22 E)$-ergosta-7,22diene-3,5,6 triol (cerevisterol) $(6)$} \\
\hline \multicolumn{13}{|c|}{ ergosta-4,6,8(14),22-tetraen-3-one (7) } \\
\hline \multicolumn{13}{|l|}{ ergosta-5-8(14),22-trien-7-one, 3 $\beta$-ol (H1-A) (8) } \\
\hline \multicolumn{13}{|l|}{$\beta$-sitosterol (9) } \\
\hline \multicolumn{13}{|l|}{ stigmasterol (10) } \\
\hline \multicolumn{13}{|l|}{ campesterol (11) } \\
\hline \multicolumn{13}{|l|}{ adenosine (12) } \\
\hline \multicolumn{13}{|l|}{ cordycepin (13) } \\
\hline \multicolumn{13}{|l|}{ cordyceamides A (14) } \\
\hline \multicolumn{13}{|l|}{ cordyceamides B (15) } \\
\hline \multicolumn{13}{|c|}{ cordycedipeptide A (16) } \\
\hline \multicolumn{13}{|c|}{ 1-(5-hydroxymethyl-2-furyl)- $\beta$-carboline (perlolyrine) (17) } \\
\hline \multicolumn{13}{|c|}{ cordymin $(\mathbf{1 8})$} \\
\hline \multicolumn{13}{|l|}{ 3',4',7-trihydroxyisoflavone (19) } \\
\hline \multicolumn{13}{|l|}{ 11,11'-dideoxyverticillin (20) } \\
\hline \multicolumn{13}{|l|}{ CS-F10 (Gal:Glc:Man = 43:33:24) (21) } \\
\hline \multicolumn{13}{|l|}{ CS-F30 (Gal:Glc:Man = 62:28:10) (22) } \\
\hline \multicolumn{13}{|l|}{ CSP-1 (Glc:Man:Gal = 1:0.6:0.75) (23) } \\
\hline cordysinocan (Glc:Man:Gal = 2.4:2.1) (24) & & & & & & & & & 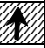 & & & \\
\hline \multicolumn{13}{|l|}{ CPS1 (Glc:Man:Gal = 2.8:2.9:1) (25) } \\
\hline \multicolumn{13}{|l|}{ PS-A (Glc:Gal:Man = 2:1:1) (26) } \\
\hline $\begin{array}{l}\text { CS-PS (Man:Rhm:Ara:Xyl:Glc:Gal = } \\
\text { 38.37:2.51:2.21:5.22:27.44:24.45) (27) }\end{array}$ & & & & & & & & & 1 & & & \\
\hline D-mannitol (28) & & & & & & & & & & & & \\
\hline butylated hydroxytouline (29) & & & & & & & & & & & & \\
\hline
\end{tabular}

*Each compound (numbered in bold inside parentheses) is followed by reference and pharmacological activities ${ }^{\mathrm{A}-\mathrm{L}}$ : (1) Zhao et al. (2009) $)^{\mathrm{K}}$, (2) Kobori et al. (2007) ${ }^{\mathrm{A}, \mathrm{C}}$, (3) Matsuda et al. (2009) $)^{\mathrm{A}}$, (4) Yang et al. (2011) ${ }^{\mathrm{C}, \mathrm{D}}$, (5) Matsuda et al. (2009) ${ }^{\mathrm{A}}$, Bok et al. $(1999)^{\mathrm{A}},(6)$ Lin et al. $(2004)^{\mathrm{G}}$, Lin $(2002)^{\mathrm{K}},\left(\text { 7) Zhao et al. }(2011)^{\mathrm{A}},(\mathbf{8}) \text { Lin et al. (1999) }\right)^{\mathrm{E}}$, Yang et al. (2003) $)^{\mathrm{I}},(9)$ Awad et al. (2009) ${ }^{\mathrm{A}}$, Zhang et al. (2011a) ${ }^{\mathrm{B}}$, Fraile et al. (2012) ${ }^{\mathrm{I}},(\mathbf{1 0})$ Gabay et al. (2010) ${ }^{\mathrm{C}}$, Ostlund (2007) $)^{\mathrm{J}}$, (11) Ostlund (2007) $)^{\mathrm{J}}$, (12) Maggirwar et al. $(1994)^{\mathrm{D}}$, Yue et al. $(2008)^{\mathrm{G}},(13)$ Matsuda et al. $(2009)^{\mathrm{A}}$, Holliday \& Cleaver $(2008)^{\mathrm{A}}$, Leu et al. $(2011)^{\mathrm{B}}$, Kim et al. $(2006)^{\mathrm{C}}$, Yang et al. $(2011)^{\mathrm{C}}$, Xiao et al. (2012) , Yun et al. , (2003) ${ }^{\mathrm{F}}$, Yue et al. $(2008)^{\mathrm{G}}$, Zhou et al. (2008) $)^{\mathrm{I}}$, Kim et al. (2009) , (14) Jia et al. (2005, 2009) ${ }^{\mathrm{A}}$, (15) Jia et al. $(2005,2009)^{\mathrm{A}}$, (16) Jia et al. $(2005)^{\mathrm{A}},(\text { 17) Yang et al. (2011) } \mathrm{C}, \mathrm{D} \text {, (18) Wang et al. (2012a) })^{\mathrm{C}, \mathrm{D}}$, (19) Yang et al. $(2011)^{\mathrm{D}}$, (20) Chen et al. (2009)A , (21) Kiho et al. (1999) ${ }^{\mathrm{E}, \mathrm{F}}$, (22) Kiho et al. (1996), (2009) $)^{\mathrm{A}, \mathrm{D}, \mathrm{I}}$, (25) Wang et al. (2009b) ${ }^{\mathrm{D}}$, (26) Kim (2010) ${ }^{\mathrm{D}, \mathrm{J}}$, (27) Zhang et al. (2011b) $)^{\mathrm{D}, \mathrm{I}}$, (28) Ohkuma et al. (1998) ${ }^{\mathrm{B}}$, Ilowite et al. $(2008)^{\mathrm{G}}$, Lameire et al. $(2003)^{\mathrm{K}}$, Rangel-Castilla et al. $(2008)^{\mathrm{L}}$, (29) Babu \& Wu (2008) $)^{\mathrm{D}}$, Jilal \& Devraj $(1996)^{\mathrm{H}, \mathrm{J}}, \downarrow$ immunosupression, $\uparrow$ immunostimulation. 


\section{Discussion}

Several pharmacological effects have been described for active fractions of $O$. sinensis which need further research for finding exact responsible metabolites. Shashidhar et al. (2013) emphasized on need of chemical characterization and mechanisms behind therapeutic effects of active components at molecular level. We have considered here only activities reported for isolated compounds and those with well defined composition. The effective nature of $O$. sinensis can be attributed to several metabolites for anti-cancer, aphrodisiac, anti-inflammation, antioxidant, hepatoprotection, anti-hypoglycemic, anti-asthemic, immunomulatory, hypolipidemic, hypocholesterolemic, renoprotective and antihypertension (Matrix 1). Among pharmacological effects, immunomodulating effect is of special mention as it can control autoimmune disorders as well as inflammation. The reports of role of $O$. sinensis in cure of autoimmune disorder by ergosta-5-8(14),22trien-7-one, $3 \hat{a}$-ol $(\mathbf{8})$ by modulation of the signal transduction proteins Bcl-2 and Bcl-XL (Yang et al. 2003) and immunosuppressive function of cordycepin (13) by increased interleukin (IL)-10 expression, decreased IL-2 expression and suppression of T lymphocyte activity leading to prevention of graft rejection in organ transplant are noteworthy (Zhou et al. 2008). There is also presence of immunostimulant compounds such as cordysinocan (24) (Cheung et al. 2009) and CS-PS (27) (Zhang et al. 2011b) in $O$. sinensis. The differential regulation of dendritic cells depending on physiological condition as identified by Li et al. (2009) or variation in metabolites produced by different strains can also be the reason of immunostimulating or immunosuppressing effects. As inflammation is also one of the hallmark of aging (Zhang et al. 2013a) and presence of compounds like ergosterol peroxide (2), ergosteryl-3-O-â-Dglucopyranoside (4), stigmasterol (10), cordycepin (13), perlolyrine (17), and cordymin (18) with the antiinflammatory property can have role in anti-ageing effect of $O$. sinensis. Cordycepin is one of the most active compound with several activities (Yun et al. 2003, Kim et al. 2006, Holliday \& Cleaver 2008, Yue et al. 2008, Kim et al. 2009, Leu et al. 2011, Yang et al. 2011, Xiao et al. 2012). There is possibility of its role in synergetic effect with other compounds for several therapeutic effects. Further, the presence of Dmannitol (Guan et al. 2010) and butylated hydroxytouline (Yu et al. 2012) in considerable amount in $O$. sinensis can be another possible reason for its acclaimed healing properties as well as vigor and vitality. The effectiveness of $O$. sinensis as infusion with other herbs also deserves attention. A mechanism for grading $O$. sinensis from different parts of Nepal and quality certification of standard is needed to give product deserved value. There are reports of isolation of several strains of Cordyceps (Singh et al. 2009, Meng et al. 2013), significant genetic differentiation (Zhang et al. 2013b) and variation in their pharmacological effect (Meng et al. 2013). Therefore, search for potential strains of Cordyceps in Himalayas with beneficial metabolites and adequate growth for commercial production are needed to provide alternative to declining wild $O$. sinensis. There should be national interest in further research for finding active components from wild $O$. sinensis, as there is still limited study of constituents based on wild resource (Zhao et al. 2013). As $O$. sinensis is revered as an epitome of Himalayan elixir due to presence of several pharmacologically active compounds with potential anti-cancer, antioxidant, immunomodulatory as well as aphrodisiac properties, the in-depth further research for scrutinizing active metabolites is anticipated.

\section{Acknowledgements}

We thank Next-Generation Bio-Green 21 Program (No. PJ0095742013), Rural Development Administration, Republic of Korea for providing research facilities.

\section{References}

Awad, A., K.C. Chan, A.C. Downie and C.S. Fink. 2009. Peanuts as a source of beta-sitosterol, a sterol with anticancer properties. Nutrition and Cancer 36: 238241.

Babu, B. and Wu, J.T. 2008. Production of natural butylated hydroxytoluene as an antioxidant by freshwater phytoplankton. Journal of Phycology 44: 1447-1454.

Baral, S.R. and P.P. Kurmi. 2006. A compendium of medicinal plants of Nepal. R. Sharma published, Kathmandu, Nepal. 534 pp.

Bok, J.W., L. Lermer, J. Chilton, H.G. Klingeman and G.H.N Towers. 1999. Antitumor sterols from the mycelia of Cordyceps sinensis. Phytochemistry 51: 891-898.

Chen, P.X., S. Wang, S. Nie and M. Marcone. 2013. Properties of Cordyceps sinensis: A review. Journal of Functional Foods 5: 550-569.

Chen, Y., H. Guo, Z. Du, X.Z. Liu, Y. Che and X. Ye. 2009. Ecology-based screen identifies new metabolites from a Cordyceps-colonizing fungus as cancer cell proliferation inhibitors and apoptosis inducers. Cell Proliferation 42: 838-847. 
Chen, Y.Q., B. Hu, F. Xu, W. Zhang, H. Zhou and L.H. Qu. 2004. Genetic variation of Cordyceps sinensis, a fruitbody-producing entomopathogenic species from different geographical regions in China. FEMS Microbiology Letters 230: 153-158.

Cheung, J.K.H., J. Li, A.W.H. Cheung, Y. Zhu, K.Y.Z. Zheng, C.W.C. Bi, R. Duan, R.C.Y. Choi, D.T.W. Lau, T.T.X. Dong, B.W.C Lau and K.W.K. Tsim. 2009. Cordysinocan, a polysaccharide isolated from cultured Cordyceps, activates immune responses in cultured T-lymphocytes and macrophages: signaling cascade and induction of cytokines. Journal of Ethnopharmacology 124: 61-68.

Devkota, S. 2006. Yarsagumba [Cordyceps sinensis (Berk.) Sacc.]; traditional utilization in Dolpa district, Western Nepal. Our Nature 4: 48-52.

Dong, C.H., Y.J. Yao 2011. On the reliability of fungal materials used in studies on Ophiocordyceps sinensis. Journal of Industrial Microbiology and Biotechnology 38: 1027-1035.

Fraile, L., E. Crisci, E. Cordoba, M.A. Navarro, J. Osada, M. Montoya. 2012. Immunomodulatory properties of Beta-sitosterol in pig immune response. International Immunopharmacology 13: 316-321.

Fu, H., W. Fu, M. Sun, Q. Shou, Y. Zhai, H. Cheng, L. Teng, X. Mou, Y. Li, S. Wan, S. Zhang, Q. Xu, X. Zhang, J. Wang, J. Zhu, X. Wang, X. Xu, G. Lv, L. Jin, W. Guo and Y. Ke. 2011. Kinetic cellular phenotypic profiling: prediction, identification, and analysis of bioactive natural products. Analytical Chemistry 83: 6518-6526.

Gabay, O., C. Sanchez, C. Salvat, F. Chevy, M. Breton, G. Nourissat, C. Wolf, C. Jacques and F. Berenbaum. 2010. Stigmasterol: a phytosterol with potential antiosteoarthritic properties. Osteoarthritis Cartilage 18: 106-116.

Gewali, M.B. 2008. Aspects of traditional medicine in Nepal. Ed. S. Awale, Institute of Natural Medicine, University of Toyama, Japan, $175 \mathrm{pp}$.

Giove, C. 2011. New Yorkers paying $\$ 800$ an ounce for worms that promise sexual prowess. NY Post, Jan 16.(http://www.nypost.com/p/news/local/ arousing success_for_love bug p4CC6laeuVGjIQ4Q7xdPTK)

Guan, J., F.Q. Yang and S.P. Li. 2010. Evaluation of carbohydrates in natural and cultured Cordyceps by pressurized liquid extraction and gas chromatography coupled with mass spectrometry. Molecules 15: 42274241.

Holliday, J. and M. Cleaver. 2008. Medicinal value of caterpillar fungi species of the Genus Cordyceps (Fr.) (Ascomycetes): a review. International Journal of Medicinal Mushrooms 10: 219-234.

Holliday, J., M. Cleaver and S.P. Wasser. 2005. Cordyceps. In: Encyclopedia of dietary supplements (Eds. P.M. Coats, M.R. Blackman, G. Cragg, M. Levine, J. Moss and J White). Marcel Decker, USA, pp. 1-13.
Huang, Y., J. Lu, B. Zhu, Q. Wen, F. Jia, S. Zeng, T. Chen, Y. Li, G. Cheng and Z. Yi. 1987. Toxicity study of fermentation Cordyceps mycelia B414. Zhongchengyao Yanjiu 10: 24-25.

Ilowite, J., P. Spiegler and S. Chawla. 2008. Bronchiectasis: new findings in the pathogenesis and treatment of this disease. Current Opinion in Infectious Diseases 21: 163-167.

Jia, J.M., H.H. Tao and B.M. Feng. 2009. Cordyceamides $\mathrm{A}$ and $\mathrm{B}$ from the culture liquid of Cordyceps sinensis (Berk.) Sacc. Chemical and Pharmaceutical Bulletin 57: 99-101.

Jia, J.M., X.C. Ma, C.F. Wu, L.J. Wu and G.S. Hu. 2005. Cordycedipeptide A, a new cyclodipeptide from the culture liquid of Cordyceps sinensis (Berk.) Sacc. Chemical and Pharmaceutical Bulletin 53: 582-583.

Jialal, I. and S. Devaraj. 1996. Low-density lipoprotein oxidation, antioxidants, and atherosclerosis: a clinical biochemistry perspective. Clinical Chemistry 42: 498506.

Kiho, T., A. Yamane, J. Hui, S. Usui and S. Ukai. 1996. Polysaccharides in fungi, XXXVI: Hypoglycemic activity of a polysaccharide (CS-F30) from the cultural mycelium of Cordyceps sinensis and its effect on glucose metabolism in mouse liver. Biological and Pharmaceutical Bulletin 19: 294-296.

Kiho, T., K. Ookubu, S. Usui, S. Ukai and K. Hirano. 1999. Structural features and hypoglycemic activity of a polysaccharide (CS-F10) from the cultured mycelium of Cordyceps sinensis. Biological and Pharmaceutical Bulletin 22: 966-970.

Kim, G.H., B. Shrestha, S.Y. Lim, D.H. Yoon, W.C. Chang, D.J. Shin, S.K. Han, S.M. Park, J.H. Park, J.M. Sung, Y. Jang, N. Chung, K.C. Hwang and T.W. Kim. 2006. Cordycepin inhibits lipopolysaccharide-induced inflammation by the suppression of NF-êB through Akt and p38 inhibition in RAW 264.7 macrophage cells. European Journal of Pharmacology 545: 192199.

Kim, H.O. and J.W. Yun. 2005. A comparative study of the production of exopolysaccharides between two entomopathogenic fungi Cordyceps militaris and Cordyceps sinensis in submerged mycelia cultures. Journal of Applied Microbiology 99: 728-738.

Kim, S.D. 2010. Isolation, structure and cholesterol esterase inhibitory activity of a polysaccharide, PS-A, from Cordyceps sinensis. Journal of Korean Society for Applied Biological Chemistry 53: 784-789.

Kim, S.G., G. Bo, S.C. Lee and H.M. Hwan. 2009. Cordycepin for inhibiting movement and proliferation of vascular smooth muscle cells. Korean Kongkae Taeho Kongbo Patent KR082739.

Kobori, M., M. Yoshida, M. Oshinishi-Kameyama and H. Shinmoto. 20007. Ergosterol peroxide from an edible mushroom suppresses inflammatory responsed in RAW254 macrophages and growth of HT29 colon 
adenocarcinoma cells. British Journal of Pharmacology 150: 209-219.

Lameire N.H., A.S. DeVriese and R. Vanholder. 2003. Prevention and nondialytic treatment of acute renal failure. Current Opinion in Critical Care 9: 481-490.

Lei, W., H. Chen, G.R. Zhang, S.S. Li, Q.Y. Peng, X. Zhong and X. Liu. 2011. Molecular identification and food source inference of constructive plants, native to the Ophiocordyceps sinensis habitat. African Journal of Biotechnology 10: 159-167.

Leu, S.F., S.L. Poon, H.Y. Pao and B.M. Huang. 2011. The in vivo and in vitro stimulatory effects of cordycepin on mouse Leydig cell steroidogenesis. Bioscience, Biotechnology, and Biochemistry 75: 723-731.

Li, C.Y., C.S. Chiang, M.L. Tsai, R.S. Hseu, W.Y. Shu, C.Y. Chuang, Y.C. Sun, Y.S. Chang, J.G. Lin., C.S. Chen, C.L. Huang and I.C. Hsu. 2009. Two-sided effect of Cordyceps sinensis on dendritic cells in different physiological stages. Journal of Leukocyte Biology 85 : 987-995.

Li, S.P., F.Q. Yang and K.W.K. Tsim. 2006a. Quality control of Cordyceps sinensis, a valued traditional Chinese medicine. Journal of Pharmaceutical and Biomedical Analysis 41: 1571-1584.

Li, S.P., G.H. Zhang, Q. Zeng, Z.G. Huang, Y.T. Wang, T.T. Dong, K.W. Tsim. 2006b. Hypoglycemic activity of polysaccharide, with antioxidation, isolated from cultured Cordyceps mycelia. Phytomedicine 13: 428433.

Li, S.P., K.J. Zhao, Z.N. Ji, Song, Z.H., T.T.X. Dong, C.K. Lo, J.K.H. Cheung, S.Q. Zhu and K.W.K. Tsim. 2003. A polysaccharide isolated from Cordyceps sinensis, a traditional Chinese medicine, protects $\mathrm{PC} 12$ cells against hydrogen peroxide-induced injury. Life Sciences 73: 2503-2513

Li, S.P., Z.R. Su, T.T.X. Dong and K.W.K. Tsim. 2002. The fruiting body and its caterpillar host of Cordyceps sinensis show close resemblance in main constituents and anti-oxidation activity. Phytomedicine 9: 319-234.

Li, T.X. and Yang, J.S. 2009. A review and prospect on the studies of Cordyceps sinensis (Berk.) Sacc. Chinese Journal of Medical Guide 11: 1310-1316.

Lie, M.H., Q.X. Zhang, M.S. Zhu, X.Y. Liang and S.Q. Li. 2005. A study of acute and subchronic toxicity of Cordyceps sinensis polysaccharides in oral liquids. Modern Preventive Medicine 32: 1062-1063.

Lin, B.Q. and S.P. Li. 2011. Cordyceps as an Herbal Drug (Chapter 5). In: Herbal medicine: biomolecular and clinical aspects (Eds. I.F.F. Benzie \& S. WactchelGalor. $2^{\text {nd }}$ edition. Boca Ration (FL), CRC Press.

Lin, C.Y. 2002. (24R)-ergosta-7,22-dien-3â,5á,6â-triol from Cordyceps sinensis for improving kidney function in renal diseases. Patent JP 2002053488 A 20020219.

Lin, C.Y., C.C. Lin, C.C. Chen and Y.C. Kuo. 2004. The isolation of active fraction and active compound from
Cordyceps sinensis can be used to improve bronchial hyper-responsiveness. Patent TW 582999 B 20040411.

Lin, C.Y., F.M. Ku, Y.C. Kuo, C.F. Chen, W.P. Chen, A. Chen and M.S. Shiao. 1999. Inhibition of activated human mesangial cell proliferation by the natural product of Cordyceps sinensis (H1-A): an implication for treatment of IgA mesangial nephropathy. Journal of Laboratory and Clinical Medicine 133: 55-63.

Liu, X., T.L. Lee and Wong, T.H. 2006. Cycloxygenase-1 inhibition shortens the duration of diazepam-induced loss of righting reflex in mice. Anesthesia and Analgesia 102: $135-140$

Matsuda, H., J. Akaki, S. Nakamura, Y. Okazaki, H. Kojima, M. Tamesada and M. Yoshikawa. 2009 Apotopsisinducing effect of sterols from the dried powder of cultured mycelium of Cordyceps sinensis. Chemical and Pharmaceutical Bulletin 57: 411-414.

Meena, H., K.P. Singh, P.S. Negi and Z. Ahmed. 2013. Subacute toxicity of cultured mycelia of Himalayan entomogenous fungus Cordyceps sinensis (Berk.) Sacc. in rats. Indian Journal of Experimental Biology 51: 381-387.

Meng, L.Z., B.Q. Lin, B. Wang, K. Feng, D.J. Hu, L.Y. Wang, K.L. Cheong, J. Zhao and S.P. Li. 2013. Mycelia extracts of fungal strains isolated from Cordyceps sinensis differently enhance the function of RAW 264.7 macrophages. Journal of Ethnopharmacology 148: 818825.

Maggirwar, S.B., D.N. Dhanraj, S.M. Somani and V. Rajkumar. 1994. Adenosine acts as an endogenous activator of the cellular antioxidant defense system. Biochemical and $B$ iophysical research Communications 201: 508-515.

MMDBD. 2012. Medicinal Materials DNA Barcode Database. http://137.189.42.34/mherbsdb/search.php

Ohkuma, S., M. Katsura, Y. Hibino, A. Hara, K. Shirotani, E. Ishikawa and K. Kuriyama. 1998. Mechanisms for facilitation of nitric oxide-evoked $\left[{ }^{3} \mathrm{H}\right] \mathrm{GABA}$ release by removal of hydroxyl radical. Journal of Neurochemistry 71: 1501-1510.

Ostlund, R.E. 2007. Phytosterols, cholesterol absorption and healthy diets. Lipids 42: 41-45.

Rangel-Castilla, L., S. Gopinath and C.S. Robertson. 2008. Management of intracranial hypertension. Neurologic Clinics 26: 521-541.

Shashidhar, M.G., P. Giridhar, K. Udaya Sankar, B. Manohar. 2013. A potent food supplement - a review. Journal of Functional Foods 5: 1013-1030.

Shen, Q.P., G.H. Qin and L.F. He. 2001. Toxicity and mutation of $C$. sinensis polysaccharide powder fed orally. Teratogenesis Carcinogenesis and Mutagenesis 13: 179-181.

Shiao, M.S., Z.N. Wang, L.J. Lin, J.Y. Lien and J.J. Wang. 1994. Profiles of nucleosides and nitrogen bases in 
Chinese medicinal fungus Cordyceps sinensis and related species. Botanical Bulletin Academia Sinica 35: 261-267.

Shrestha, B., W.M. Zhang, Y.J. Zhang and X.Z. Liu. 2010. What is the Chinese caterpillar fungus Ophiocordyceps sinensis (Ophiocordycipitaceae)? Mycology 1: 228236.

Shrestha, S., B. Shrestha, J.H. Park, D.Y. Lee, J.G. Cho, D.G. Lee and N.I. Baek. 2012. Chemical Constituents of Yarsagumba (Ophiocordyceps sinensis (Berk.) Sung et al.), a Valued Traditional Himalayan Medicine. Nepal Journal of Science and Technology 13: 43-58.

Singh, R., P.S. Negi, Z. Ahmed. 2009. Genetic variability assessment in medicinal caterpillar fungi Cordyceps spp. (Ascomycetes) in Central Himalayas, India. International Journal of Medicinal Mushrooms 11: 185189.

Sung, G.H., N.L. Hywel-Jones, J.M. Sung, J.J. Luangsaard, B. Shrestha and J.W. Spatafora. 2007. Phylogenetic classification of Cordyceps and clavicipitaceous fungi. Studies in Mycology 57: 5-59.

Wang, J., Y.M. Liu, W. Cao, K.W. Yao, Z.Q. Liu and J.U. Guo. 2012a. Anti-inflammation and antioxidant effect of Cordymin, a peptide purified from the medicinal mushroom Cordyceps sinensis, in middle cerebral artery occlusion-induced focal cerebral ischemia in rats. Metabolic Brain Disease 27: 159-165.

Wang, S., F.Q. Yang, K. Feng, D.Q. Li, J. Zhao and S.P. Li. 2009a. Simultaneous determination of nucleosides, myriocin, and carbohydrates in Cordyceps by HPLC coupled with diode array detection and evaporative light scattering detection. Journal of Separation Science 32: 4069-4076.

Wang, X.B., Y.Y. Jiang and C.Y. Zhao. 2012b. Clinical research of xinganbao capsule on the treatment of chronic hepatitis B liver fibrosis. Chinese Journal of Integrated Traditional and Western Medicine 32: 325328.

Wang, X.L. and Y.J. Yao. 2011. Host insect species of Ophiocordyceps sinensis: a review. ZooKeys 127: 4359.

Wang, Y., M. Wang, Y. Ling, W. Fan, Y. Wang and H. Yin. 2009b. Structural determination and antioxidant activity of a polysaccharide from the fruiting bodies of cultured Cordyceps sinensis. The American Journal of Chinese Medicine 37: 977-989.

Xiao, L., Y. Ge, L. Sun, X.X. Xu, P. Xie, M. Zhan, M. Wang, Z. Dong, J. Li, S. Duan, F. Liu and P. Xiao. 2012. Cordycepin inhibits albumin-induced epithelialmesenchymal transition of renal tubular epithelial cells by reducing reactive oxygen species production. Free Radical Research 46: 174-183.

Yang, L.Y., W.J. Huang, H.G. Hsieh, C.Y. Lin. 2003. H1-A extracted from Cordyceps sinensis suppresses the proliferation of human mesangial cells and promotes apoptosis, probably by inhibiting the tyrosine phosphorylation of Bcl-2 and Bcl-XL. Journal of Laboratory and Clinical Medicine 141: 74-83.

Yang, M.L., P.C. Kuo, T.L. Hwang and T.S. Wu. 2011. Anti-inflammatory principles from Cordyceps sinensis. Journal of Natural Products 74: 1996-2000.

Yu, S.J., Y. Zhang and M.Z. Fan. 2012. Analysis of volatile compounds of mycelia of Hirsutella sinenis, the anamorph of Ophiocordyceps sinensis. Applied Mechanics and Material 140: 253-257.

Yue, G.G.L, C.B.S. Lau, K.P. Fung, P.C. Leung and W.H. Ko. 2008. Effects of Cordyceps sinensis, Cordyceps militaris and their isolated compounds on ion transport in Calu-3 human airway epithelial cells. Journal of Ethnopharmacology 117: 92-101.

Yun, Y.H., S.H. Han, S.J. Lee, S.K. Ko, C.K. Lee, N.J. Ha and K.J. Kim. 2003. Anti-diabetic effects of CCCA, CMKSS, and cordycepin from Cordyceps militaris and the immune responses in streptozotocin-induced diabetic mice. Natural Product Sciences 9: 291-298.

Zhang, G., L. Juxue, S. Purkayastha, Y. Tang, H. Zhang. 2013a. Hypothalmic programming of systemic ageing involving IKK- $\beta$, NF- $\kappa$ B and GnRH. Nature 497: 211 216.

Zhang, J., J. Hao, J. Guo, B. He and H. Huang. 2011a. Effect of fistular onion stalk extract on the level of NO and expression endothelial NO synthase (eNOS) in human umbilical vein endothelium cells. African Journal of Biotechnology 10: 2536-2540.

Zhang, J., Y. Yu, Z. Zhang, Y. Ding, X. Dai and Y. Li. 2011 b. Effect of polysaccharide from cultured Cordyceps sinensis on immune function and anti-oxidation activity of mice exposed to ${ }^{60} \mathrm{Co}$. International Immunopharmacology 11: 2251-2257.

Zhang, S., Y.J. Zhang, X.Z. Liu, H. Zhang and D.S. Liu. $2013 \mathrm{~b}$. On the reliability of DNA sequences of Ophiocordyceps sinensis in public databases. Journal of Industrial microbiology and Biotechnology 40: 365378.

Zhao, J., J. Xie and L.Y. Wang. 2013. Advanced development in chemical analysis of Cordyceps. Journal of Pharmaceutical and Biochemical Analysis http:// dx.doi.org/10.1016/j.jpba.2013.04.025

Zhao, Y.Y., X. Shen, X. Chao, C.C. Ho, X.L. Cheng, Y. Zhang, R.C. Lin, K.J. Du, W.J. Luo, J.Y. Chen and W.J. Sun. 2011. Ergosta-4,6,8(14),22-tetraen-3-one induces $\mathrm{G} 2 / \mathrm{M}$ cell cycle arrest and apoptosis in human hepatocellular carcinoma HepG2 cells. Biochimica et Biophysica Acta - General Subjects 1810: 384-390.

Zhao, Y.Y., R.M. Xie, X. Chao, Y. Zhang, R.C. Lin and W.J. Sun. 2009. Bioactivity-directed isolation, identification of diuretic compounds from Polyporus umbellatus. Journal of Ethonopharmacology 126: 184-187. 
S. Shrestha et al./A Brief Review on Pharmacologically.....

Zhou, X., L. Luo, W. Dressel, G. Shadier, D. Krumbiegel, P. Schmidtke, F. Zepp, C.U. Meyer. 2008. Cordycepin is an immunoregulatory active ingredient of Cordyceps sinensis. The American Journal of Chinese Medicine 36: 967-980.

Zhu, J.S., G.M. Halpern and K. Jones. 1998a. The scientific rediscovery of an ancient Chinese herbal medicine
Cordyceps sinensis: Part I. Journal of Alternative and Complementary Medicine 4: 289-303.

Zhu, J.S., G.M. Halpern and K. Jones. 1998b. The scientific rediscovery of a precious ancient Chinese herbal regimen Cordyceps sinensis: Part II. Journal of Alternative and Complementary Medicine 4: 429-457. 
Nepal Journal of Science and Technology Vol. 14, No. 2 (2013) 49-58 